\title{
Avoiding pitfalls in shallow seismic reflection surveys
}

\author{
Don W. Steeples* and Richard D. Miller ${ }^{\ddagger}$
}

\begin{abstract}
Acquiring shallow reflection data requires the use of high frequencies, preferably accompanied by broad bandwidths. Problems that sometimes arise with this type of seismic information include spatial aliasing of ground roll, erroneous interpretation of processed airwaves and air-coupled waves as reflected seismic waves, misinterpretation of refractions as reflections on stacked common-midpoint (CMP) sections, and emergence of processing artifacts. Processing and interpreting nearsurface reflection data correctly often requires more than a simple scaling-down of the methods used in oil and gas exploration or crustal studies. For example, even under favorable conditions, separating shallow reflections from shallow refractions during processing may prove difficult, if not impossible. Artifacts emanating from inadequate velocity analysis and inaccurate static corrections during processing are at least as troublesome when they emerge on shallow reflection sections as they are on sections typical of petroleum exploration. Consequently, when using shallow seismic reflection, an interpreter must be exceptionally careful not to misinterpret as reflections those many coherent waves that may appear to be reflections but are not. Evaluating the validity of a processed, shallow seismic reflection section therefore requires that the interpreter have access to at least one field record and, ideally, to copies of one or more of the intermediate processing steps to corroborate the interpretation and to monitor for artifacts introduced by digital processing.
\end{abstract}

\section{INTRODUCTION}

Problems in collecting, processing, and interpreting seismic data can impede the practical implementation of near-surface, common-midpoint (CMP), seismic reflection techniques, also known as common depth-point (CDP) methods. However, when used correctly, these same methods can be very effective in analyzing the geology of the near surface.

Detecting shallow reflectors requires the use of high frequencies, a fact first recognized by Evison (1952). For example, when a synthetic $100-\mathrm{Hz}$ source wavelet is introduced into a $10-\mathrm{m}-$ thick layer over a half-space, the first arrival separates nicely from the reflection at geophone offsets of less than $5 \mathrm{~m}$ (Figure 1). However, when a $40-\mathrm{Hz}$ dominant-frequency wavelet is used, interference between the first arrival and the reflection wavelet occurs at all offsets, including zero. Consequently, we can obtain the desired shallow reflection result only when we use high frequencies, no matter what the bandwidth. Based on this finding and on the fact that clean Ricker wavelets do not occur in real data, any event occurring earlier than $\sim 25 \mathrm{~ms}$ on a stacked section having a dominant frequency of less than $\sim 100 \mathrm{~Hz}$ cannot be considered an uncontaminated reflection.

Other problems, which we collectively term "pitfalls," involve the spatial aliasing of ground roll, interpreting airwaves and air-coupled waves as actual seismic waves, incorrectly interpreting refractions as reflections on stacked CMP sections, and failure to deal with processing artifacts. Examples of these pitfalls are grouped into sections dealing with the collection, processing, and interpretation of near-surface data, along with recommendations for either diagnosing and rectifying or avoiding the problems in each of these areas.

\section{Data-collection pitfalls}

Field testing.-Before a seismic reflection survey is undertaken at a new site, we recommend that on-site testing be done. Ideally, a full day of walkaway noise testing, a procedure in which geophones are moved progressively farther from the shotpoint, should be scheduled at the beginning of a project. We advocate testing at least two types of seismic sources (e.g., weight drops, explosives, projectiles, or Vibroseis ${ }^{\mathrm{TM}}$ ) in at least two sizes (e.g., 12- and 20-lb sledgehammers, 8 - and 12-gauge downhole shotguns) or sweep strategies per source for each project. Generally, we have found that high-quality, $40-\mathrm{Hz}$ geophones are adequate for this type of testing. However, at locations exhibiting dominant frequencies of 400 to $600 \mathrm{~Hz}$, we use $100-\mathrm{Hz}$ geophones.

Manuscript received by the Editor April 25, 1997; revised manuscript received December 1, 1997.

*Dept. of Geology, The University of Kansas, Lawrence, Kansas 66045-2124. E-mail: steeples@kuhub.cc.ukans.edu. $\ddagger$ Kansas Geological Survey, 1930 Constant Avenue, Lawrence, Kansas 66047-3726. E-mail: rmiller@kgs.ukans.edu.

(C) 1998 Society of Exploration Geophysicists. All rights reserved. 
To some degree, the relative amplitudes of reflections, as compared to ground roll, are a function of geophone coupling to the ground. In most circumstances, the best coupling is obtained when geophones mounted on long spikes are planted firmly in the earth. When the geophones are poorly coupled to the ground, ground roll is enhanced relative to reflections (see Hoover and O'Brien, 1980; Krohn, 1984).

For the purposes of walkaway testing, we have found it advisable to begin with geophone station spacings that are about half those we would expect to use for a production survey at a given site. At this stage, we also use shot-to-geophone maximum offsets that are at least $1 \frac{1}{2}$ times the depth of the primary target. Normally, we have a general set of parameters in mind when we arrive at a particular site. However, the results of the walkaway tests often require us to alter one or more of those parameters as we begin the production phase of the survey.

When using a seismograph with 16 or fewer bits of analogto-digital (A/D) conversion, we have found it advantageous to have a large selection of pre-A/D filter combinations available so we can test at least three filter settings for each walkaway test. When the seismograph has 18 bits or more of A/D conversion, we advise recording shallow reflection data without using analog, low-cut filters to avoid phase distortion and potential loss of bandwidth. However, the adequacy of the instantaneous dynamic range of the seismograph must be the overriding consideration when determining whether pre-A/D, low-cut filters are to be used. The desired data are lost when seismograph gain is too low to record at least one or two bits of reflection information, a situation that may occur when too much lowfrequency surface-wave information is allowed to reach the A/D converters. Alternatively, when gain is too high and the data are clipped, reflection data will be recorded ambiguously, in most cases rendering them useless.

\section{Geophone Offset (m)}
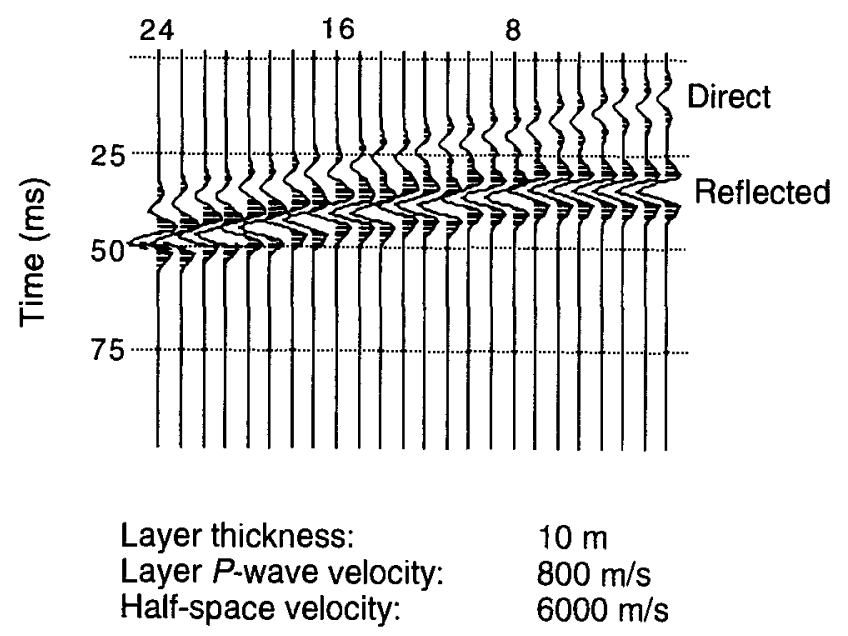

FiG. 1. A layer-over-half-space model showing $100-\mathrm{Hz}$ arrivals for the direct wave only and the primary reflection from a reflector at a depth of $10 \mathrm{~m}$. Note the interference that occurs at distances as small as $5 \mathrm{~m}$ from the shotpoint. When the dominant frequency of the data can be increased, the interference problem can be decreased.
Ground roll.--Usually, ground roll is characterized by low frequency, low velocity, and high amplitude. Because ground roll tends to mask reflection signals, it has presented a dilemma, since the 1920s, for seismologists in pursuit of hydrocarbons. Classically, the methods used to deal with ground roll are source and geophone arrays, frequency filtering, $f-k$ filtering, and stacking. Generally, however, ground roll is less troublesome during shallow surveys than it is during surveys undertaken at greater depths. Although we have performed shallow reflection surveys at more than 100 sites, including areas characterized by volcanics at or near the surface and locations with water tables $50 \mathrm{~m}$ or more below the surface, we have not yet encountered a site at which the reflections in the upper $100 \mathrm{~ms}$ did not have a dominant frequency nearly double that of the ground roll. Although such sites may exist, to date we have not found any shallow reflections with low dominant frequencies relative to the dominant frequency of the ground roll. Hence, we have been able to decrease the amplitude of the ground roll either by frequency filtering or by using a combination of frequency filtering, spectral balancing, and $f-k$ filtering. Minimizing ground roll by applying digital filtering presupposes that the instantaneous dynamic range of the equipment is large enough to allow the reflected energy to be recorded in the presence of high-amplitude noise. However, even when using systems featuring quiet electronics and 16 bits or more of $A / D$ conversion, reflected energy cannot always be recorded successfully. Alternatively, when the reflections can be maintained in the optimum window (Hunter et al., 1984), ground roll can be removed by surgical muting.

When data frequency is too low for shallow reflections.--In shallow surveying, a lower limit of acceptable reflection frequencies exists, based on seismic velocity and target depth. Often, this limit can be determined by using forward modeling, as shown in Figure 1, or by making some simple calculations. For example, assume you are trying to record shallow reflections at a time of $25 \mathrm{~ms}$ with a field situation that produces $40-\mathrm{Hz}$ dominant-frequency wavelets. Assume further that the first arrival comes in at $5 \mathrm{~ms}$ on the nearest field-file trace, which would not be uncommon in a survey so near the surface. Note that, in this example, the trailing edge of the first-arrival wavelet would not disappear until after the 30 -ms mark on the nearoffset traces. This results from adding the period of a $40-\mathrm{Hz}$ wavelet $(25 \mathrm{~ms})$ to the $5 \mathrm{~ms}$ for the first arrival. Hence, any energy appearing on the stacked sections with a time of less than $30 \mathrm{~ms}$ would be contaminated by first-arrival waves. Under these circumstances, we would not be able to engender any useful reflections earlier than $30 \mathrm{~ms}$. Also, the first arrivals tend to be somewhat ringy, so the $40-\mathrm{Hz}$ dominant-frequency wavelet specified in the example would interfere even after $30 \mathrm{~ms}$. Hence, a few additional milliseconds must pass before reflection information can be recorded without interference.

To address the lower frequency limit, we have tried numerous processing techniques, including $\tau-p$ and $f-k$ filtering, to separate direct waves and refractions from genuine shallow reflections. Of these, the only method that appears to work consistently is to generate and record much higher frequencies than those commonly used in reflection surveys involving the deeper regions. At some locations, we have not been able to work at frequencies high enough to separate shallow reflections 
from refractions and direct waves. In such cases, the seismic reflection method must be considered inappropriate in its present stage of development.

Spatial and temporal aliasing.-Aliasing occurs when data are not sampled often enough in time or space. For example, the buggy wheels that we sometimes see in Western movies frequently appear to be turning backward, even though we know they are moving forward. This phenomenon occurs because a movie camera is not designed to sample the viewing field often enough to represent what is happening in reality. Similarly, when the seismic wavefield is not sampled often enough in time, seismograms may provide a false picture of ground motion.

Modern engineering seismographs have been designed to circumvent temporal aliasing by means of antialias, high-cut filters installed at the factory. Some of these seismographs automatically select and activate an appropriate antialiasing filter before any data are recorded. Others require adjusting the seismograph manually each time the sampling rate is changed. The problems inherent in temporal aliasing and the solutions to them are generally well known to those in the fields of seismology and digital signal processing (e.g., Yilmaz, 1987), so we will not address them here.

Spatial aliasing issues, in which data are not sampled frequently enough in space, are less familiar. One example of spatially aliased shallow seismic data appears in Steeples and Miller (1990), and another is presented in Figure 2. Despite

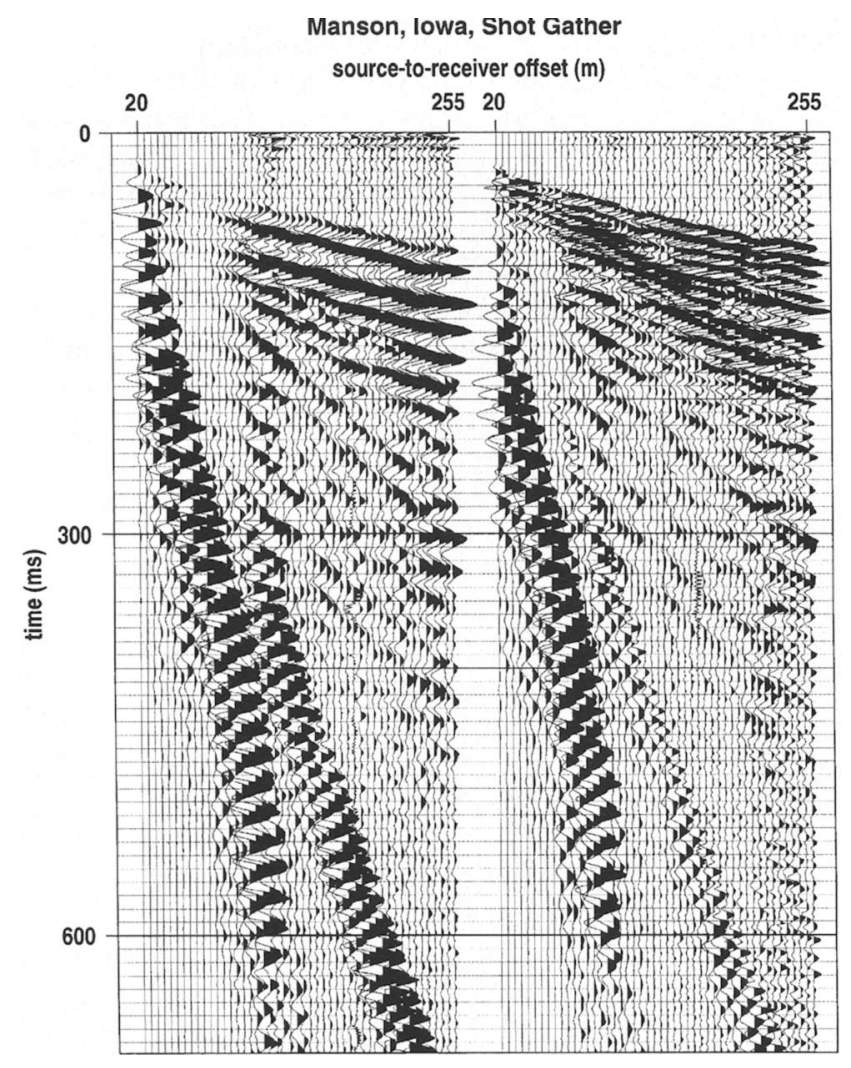

FIG. 2. Spatially aliased ground roll from the Manson impact structure, Iowa. Note the coherency of the ground roll with apparent moveout in the opposite direction from that of the first arrival. Note also that the air blast is spatially aliased (data from Keiswetter, 1995). a geophone group interval of $5 \mathrm{~m}$, the ground roll is spatially aliased, as shown by the apparent phase velocity of the ground roll in a direction opposite to that of the first arrivals. Over the past decade, the authors have had the opportunity to observe the spatial aliasing of ground roll at several sites. In theory, a seismologist might use spatially aliased data-conceivably constructing an entire survey around them-only to wonder why the "reflector" disappears during processing or, worse, why the interpretation of the data is inconsistent with the results of drilling. In fact, we are aware of one incident in which a geophysical contractor plotted aliased, common-offset ground roll as though it were an interpretable reflection. To help avoid such pitfalls, we have developed these strategies:

1) When a coherent event on a field-file seismogram is a true reflector, moving the shotpoint a major fraction of one geophone interval closer to (or farther away from) the geophone spread will have little effect on the appearance of the reflector. However, when the coherent event is the spatial aliasing of ground roll, the effect is often substantial. Figure 3 illustrates a case in which moving the shotpoint by only $1 \mathrm{~m}$ affected the seismogram substantially. The events observed at 120 and $160 \mathrm{~ms}$ initially appeared to be reflections. However, when the shotpoint was moved, a substantial change in the seismogram resulted, thus removing the coherency of both events.

2) Decreasing the geophone interval by a substantial amount, such as a factor of two or three, will improve the coherency of a true reflector but will destroy that of spatially aliased ground roll.

3) When additional geologic or geophysical information is available from a site (e.g., uphole traveltime, depth to bedrock, acoustic or other geophysical logs), a pocket calculator program (Steeples and Miller, 1990) used to calculate a least-squares-fit hyperbola to a set of $T$ - and $X$-points measured directly from a field-file seismogram may provide some assistance in assessing whether a suspected reflection is genuine.

4) Energy reflected from layers near the surface should have a frequency content close to that of the direct wave and the early refracted arrivals on the field files. When the observed reflection frequencies on field files, common-offset sections, or CMP sections are considerably lower than those of the first arrivals, the recorded events are likely to be caused by ground roll rather than by reflections.

\section{Data processing pitfalls}

Air-coupled waves.-Most seismic sources emit air-blast noise that can be heard by a bystander. When this noise couples to the ground or to geophones planted in the ground, the emissions are termed air-coupled waves. Collectively, such waves are known as airwaves. Airwaves can cause enormous problems during the processing of shallow reflection data. Our experience has been that when low-cut filters (either analog or digital) of $200 \mathrm{~Hz}$ or higher are used, airwaves commonly show a dominant frequency near that of the lower band edge of the low-cut filter used to refine the data. When low-cut filtering is minimal, the dominant frequency of the airwaves is often in the range of $150 \mathrm{~Hz}$ or higher. Simple frequency filtering does little 
to attenuate airwaves within the frequency range of reflected energy. Therefore, we prefer to use a surgical muting window to remove airwaves. The recorded air blast usually exhibits some energy at frequencies between 200 and $500 \mathrm{~Hz}$, so these waves are likely to be spatially aliased unless the geophone interval is substantially less than $1 \mathrm{~m}$. Because of spatial aliasing, $f-k$ filtering must be used with caution to avoid enhancing those components of the air-coupled waves that could stack coherently on CMP sections. In most cases air-coupled waves last several cycles, which causes a portion of the high-frequency wave to fall outside the mute window in $f-k$ space. These parts of the airwave may stack coherently at velocities that are reasonable for near-surface materials. The problem becomes more dramatic when data are scaled or trace-balanced after filtering.

Figure 4 provides an example of a CMP seismic section exhibiting what appear to be coherent reflections at 60 to $70 \mathrm{~ms}$. These are not true reflections but are stacked air blast. Figure 5 is an example of an air-blast echo from the recording truck superimposed on an intra-alluvial reflection. Note that the position of the diffraction-like pattern changed when the recording truck was moved.

In one high-resolution seismic reflection survey of which we are aware, about a dozen "reflections" were observed on a section that had been processed by experienced professionals. However, close inspection later revealed that all of the "reflections" had a dominant period of about $9 \mathrm{~ms}$, from times of $80 \mathrm{~ms}$ to $600 \mathrm{~ms}$. Furthermore, these apparent reflections had the highest $\mathrm{S} / \mathrm{N}$ ratio where the CMP fold was smallest. The S/N ratio of reflections on CMP-stacked sections should increase proportionally to the square root of the fold rather than decrease with increasing fold. So, when the data were reprocessed with appropriate surgical muting of the air blast, no usable reflections were found. Indeed, it became evident that the entire geologic interpretation was based on the CMP stacking of air blast, complete with sand lenses whose presence was by chance supported by the drilling data.

Refractions.-Separating shallow reflections from shallow refractions on field files prior to or during processing is of- ten a formidable task. We know that energy reflected near the surface tends to have a frequency content close to that of the direct wave and the early refracted arrivals on field files. As a result, reflections cannot be separated easily from refractions by means of frequency filtering. Moreover, wide-angle shallow reflections and shallow refractions have very similar phase velocities, and they may interfere with each other-destructively or constructively - over a large range of geophone offsets. Consequently, using $f-k$ filtering to separate overlapping events having similar phase velocities is ineffective because of the side-lobe effects of the filters.

Distinguishing refractions from reflections on field files can be difficult, but separating shallow reflections unequivocally from shallow refractions on processed sections without referring to the field files is nearly impossible. Even so, one useful indicator on a processed seismic section is frequency inversion with depth. Figure 6 is an example of stacked refractions that have an apparent frequency lower than that of the underlying reflections. Under normal geologic conditions, the frequency of the reflections on a shallow reflection seismogram will decrease with depth as a result of attenuation with increasing travelpath length. When the apparent frequency of the coherent events changes from low at short reflection times to high at longer times, shallow refractions may have been stacked into the data. Another possibility is that high-frequency airwaves have stacked coherently at the longer times.

In a CMP processing scheme, the configuration of a reflection-time display will be transformed from a hyperbola to a straight line during NMO correction. True shallow reflections typically lose $20-30 \%$ of their high-frequency information during this process because of NMO stretch (Miller, 1992). Refractions are stretched by a similar amount. Yet refractions at close offsets that arrive linearly do not respond in-phase to a hyperbolic transformation; thus, the misalignment of individual wavelets present during CMP stacking may drastically reduce their apparent dominant frequency. To illustrate this point, Figure 7 presents a low-frequency refraction between 20 and $40 \mathrm{~ms}$ that was allowed to remain in the data throughout the stacking process.

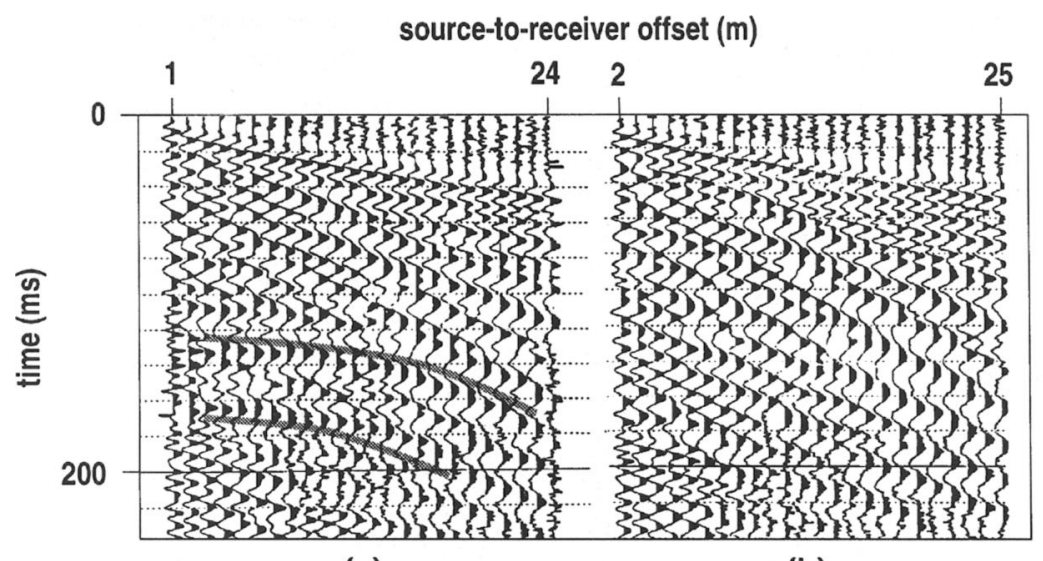

(a)

(b)

spatially aliased ground roll

FIG. 3. Possible reflections observable at 120 and $170 \mathrm{~ms}$ in the field file on the left disappear when the sledgehammer shotpoint is moved only $1 \mathrm{~m}$. Geophone plants and other parameters are the same in the field file on the right, except that the sledgehammer source was moved $1 \mathrm{~m}$ farther from the line. The apparent reflections in the field file on the left are spatially aliased ground roll. (Data collected near London, England, by R. Huggins, R. Miller, and D. Steeples, 1989.) 


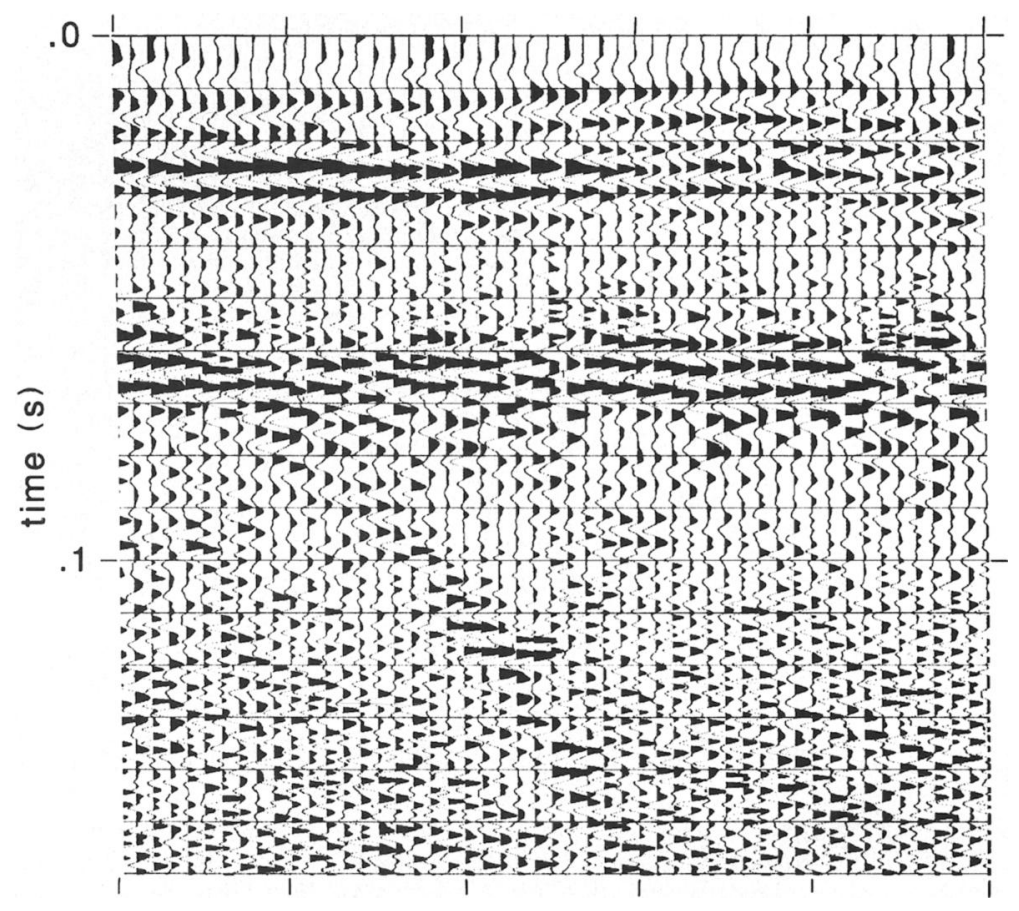

FIG. 4. Air blast appearing on stacked sections from Heber City, Utah, 12-fold CDP data. The coherent event at times between 60 and $70 \mathrm{~ms}$ is air blast (from Steeples and Miller, 1990).

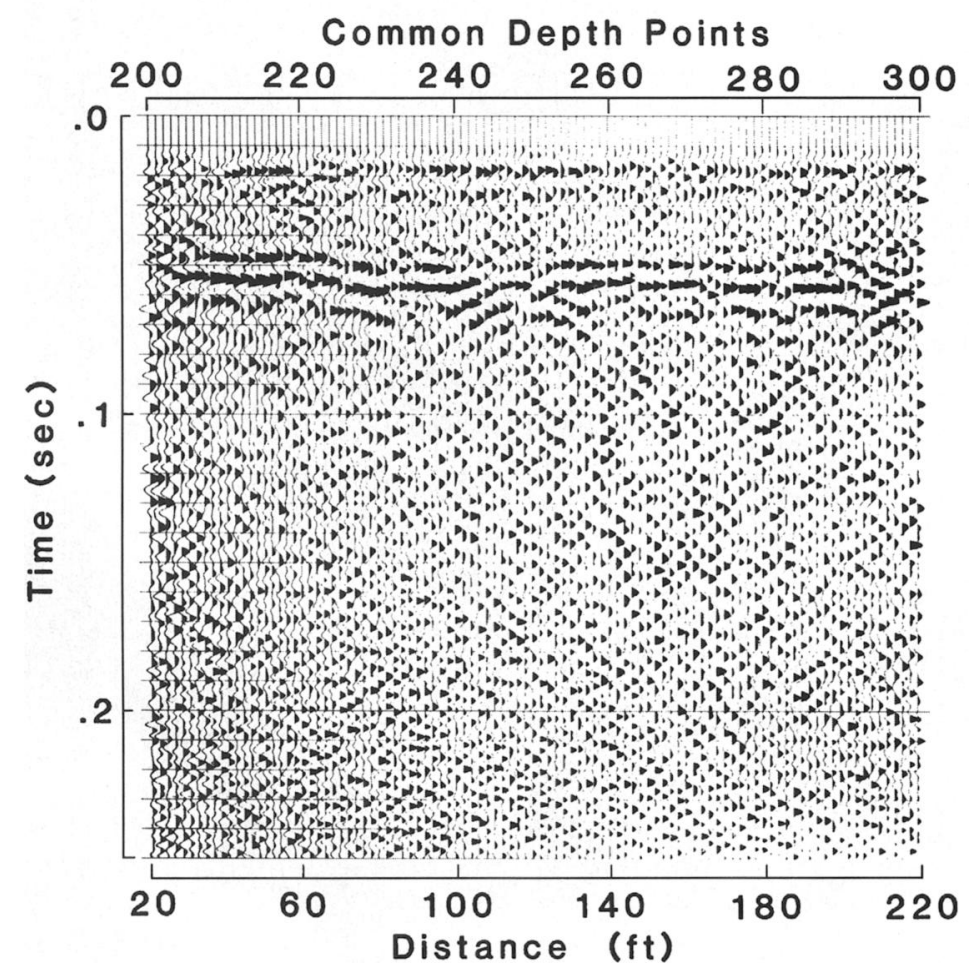

FIG. 5. An intra-alluvial reflection from the Blue River Valley, Kansas, showing the effect of improper first-arrival mute on a 12-fold CDP stack with air-blast echo from a recording truck at CDPs of 246 and 298. The air-blast echo could be mistaken for a diffraction. In reality, the truck was moved from one location to another as the survey progressed. Problems resulting from air blast that stacks constructively occur in many cases (from Steeples and Miller, 1990). 


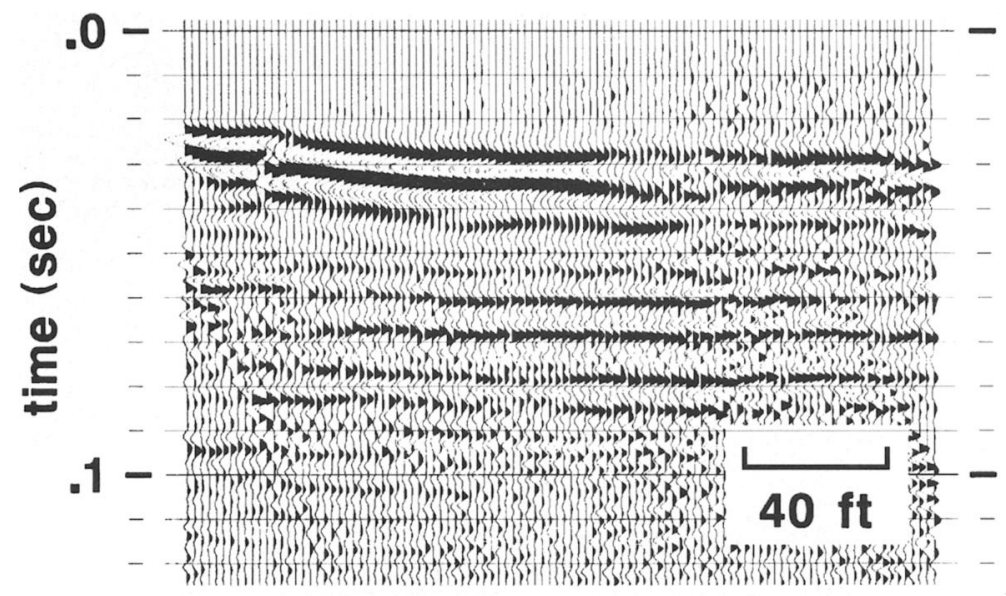

\section{Low-Cut Filter (pre A/D): $220 \mathrm{~Hz}$}

FIG. 6. An example of the effect of allowing first arrivals to remain in seismic reflection data. Energy arriving at times of less than $40 \mathrm{~ms}$ is primarily refraction energy. Note the lower apparent frequency and the apparent dip. The true reflections at times between 50 and $100 \mathrm{~ms}$ show higher frequency and a flat structure. The scale distance of $40 \mathrm{ft}$ corresponds to about $12 \mathrm{~m}$ (from Steeples and Miller, 1990).

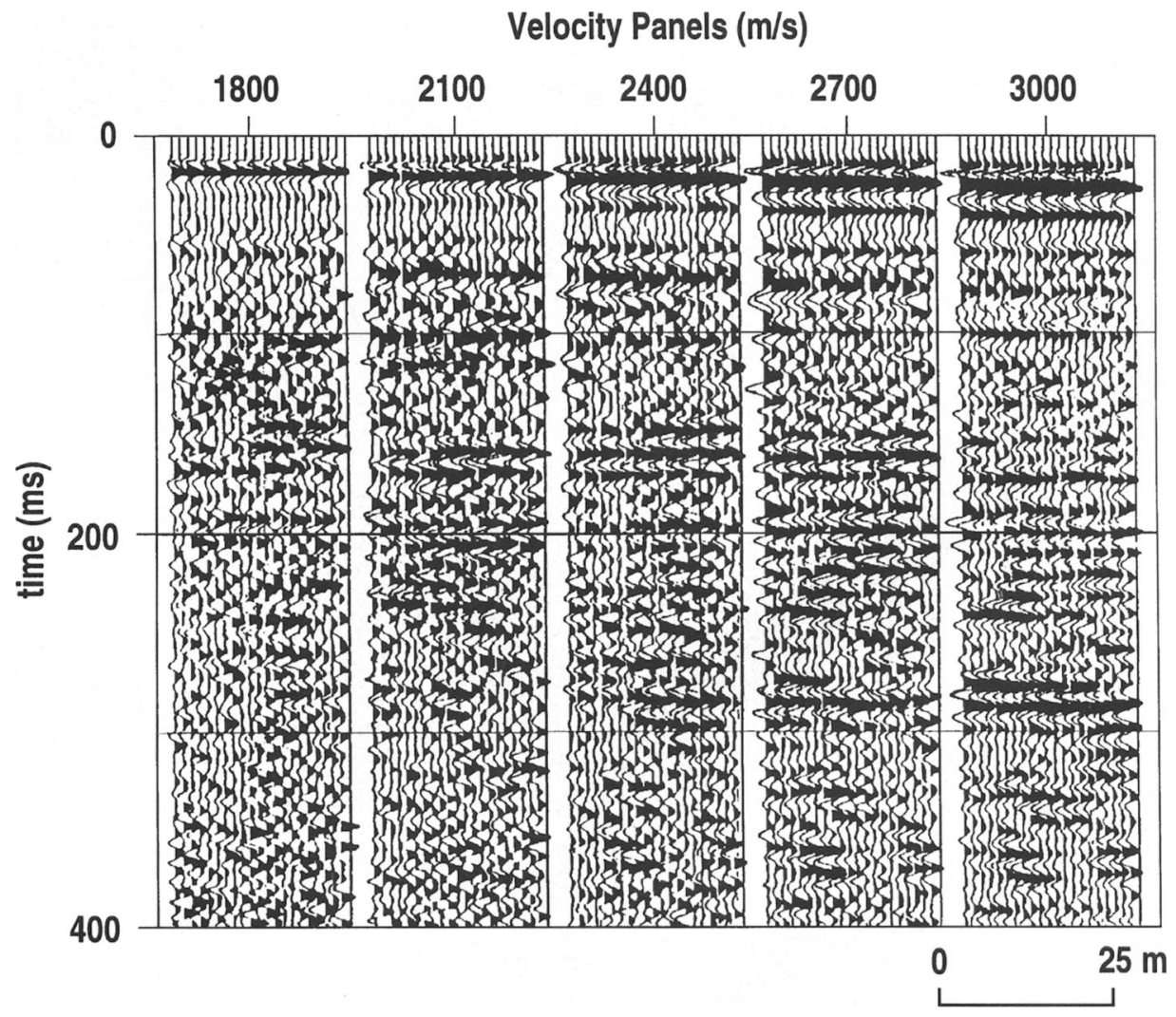

FIG. 7. Stacked velocity panels in which the first-arrival wavelets were left in the data on purpose to illustrate stacking of refractions. Velocity analysis reveals coherency at different depths for different stacking velocities. Hence, the interpretation could depend upon which velocity the processor used. 
So far, our discussion of refractions has involved flat-lying layers only. Figure 8 is an example of improperly muted refractions in a data set that includes dipping layers near the surface. As the survey progressed downdip, the depth to the refractors and the associated critical-offset refraction distance increased. At some point, the geophones were no longer farther than the critical-offset distance and the refractions disappeared. Meanwhile, signal from a shallower refractor was picked up by the geophones, and the refraction data stacked coherently for some distance along the line as the process repeated itself. The effect was a shingled appearance of the seismic record, with the stacked refractors resembling successive layers of roofing shingles.

Although misidentifying a refraction as a reflection is a common pitfall, the opposite mistake can be made as well, i.e., a genuine reflection may be discarded simply because it looks like a refraction. One way to avoid such an error is to make time-distance measurements using a field-file record and to solve the problem as though the arrival in question were a refraction. This can be accomplished by measuring the velocities and intercept times of the direct-wave first arrivals and the refraction/reflection in question. These measurements can then be introduced into the basic refraction equations for one layer over a half-space and solved for critical offset distance. If an essential contradiction develops, i.e., if the critical offset distance is much greater than the offset distance at which the phase is observed, a reflection is likely because a refraction cannot occur at distances smaller than the critical distance. When two or more layers are present over a half-space, multilayer refraction calculations can be used to observe whether an essential contradiction develops in the refraction assumption. An additional aid to resolving the reflection/refraction quandary emerges when the apparent structure of a stacked section closely resembles the topography of the surface. Although such mimicking is geologically possible, it may suggest the presence of refractions, erroneous static corrections, or an incorrect near-surface velocity model.

For quality control, the data may be processed with the direct waves and the refractions left intact within the data set so the processor can see where they emerge and what effect they may have on the processed sections. The results then can be used as a diagnostic tool to make certain that refractions, or their vestiges, are not present on the final processed section to which surgical muting has been applied. The refracted arrivals can be muted carefully, field file by field file, during the early stages of processing to reduce the likelihood of their stacking on the section. Sometimes only a small percentage of field records will show unusually good reffections, but these records can be used to correlate to the processed seismic sections.

Perhaps unfortunately, the geologic conclusions reached by assuming that an event on a stacked section is a reflection may approximate the true geology even when the event is a refraction. Greater depth to a velocity discontinuity leads to increased refractor intercept times and increased two-way reflection times; hence, the stacking of refraction arrivals with CMP reflection software can sometimes lead to a correct geologic interpretation based on either an incorrect or an inappropriate geophysical analysis. Indeed, one of the principal limitations of the shallow seismic reflection method continues to be its inability to separate, with certainty, shallow reflections from shallow refractions and guided waves.

Processing artifacts.-Artifacts that arise from inadequate velocity analysis, inaccurate static corrections, or inappropriate processing are at least as troublesome when they appear on shallow reflection sections as they are when they occur in deeper petroleum exploration reflection surveys. The deconvolution of shallow reflection data often, but not always, degrades the quality of the reflections. Usually, one or more of the theoretical assumptions underlying deconvolution (Yilmaz, 1987) is violated when dealing with shallow data. First, the highfrequency components of the source wavelet often change substantially as near-surface conditions change. Consequently, the source wavelet may change with horizontal location, which violates one of the main assumptions of spiking deconvolution. Second, the frequency of shallow reflections often varies rapidly with depth in the earth, a problem that can be addressed at least partially by using time-varying deconvolution. Third, shallow reflection data commonly show too few refiective horizons (sometimes only one or two) to represent a random reflectivity series, which is one of the primary assumptions of statistics-based deconvolution methods. Finally, the

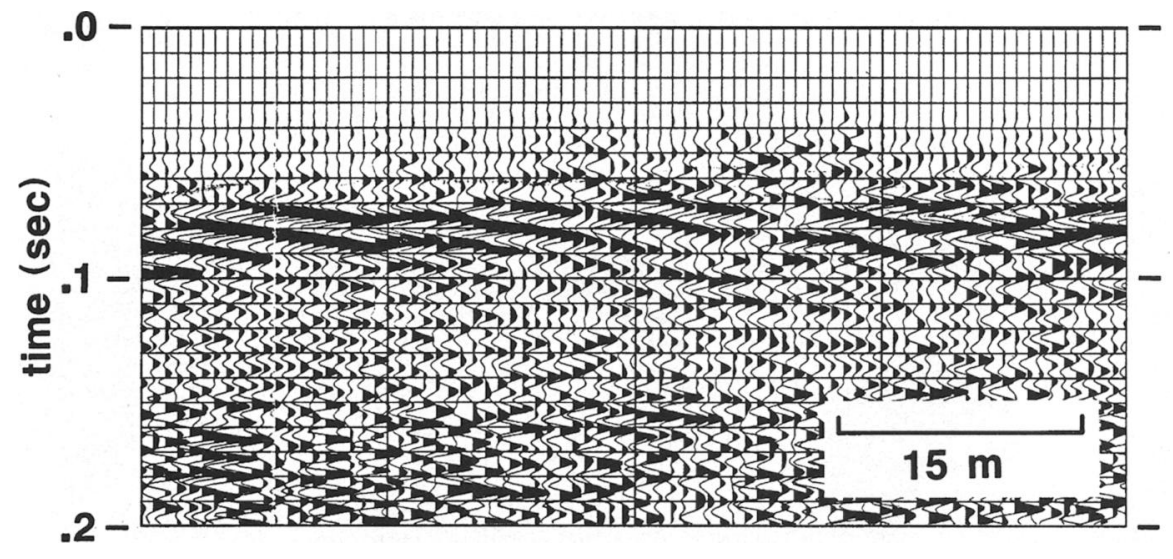

FIG. 8. An example of refractions stacking constructively when first arrivals were muted improperly. Note the shingled appearance of the coherent events between 70 and $100 \mathrm{~m} / \mathrm{s}$. This represents improperly muted refractions in a dipping environment. As the refractors dipped below the surface, their critical distance became greater than the farthest geophone offsets. Consequently, the events die out at times greater than $100 \mathrm{~m} / \mathrm{s}$ on the stacked sections. In dipping environments, such shingling is diagnostic of refractions on stacked "reflection" sections. 
$\mathrm{S} / \mathrm{N}$ ratio of shallow reflection data sets is notoriously poor, a condition exacerbated by the use of deconvolution (House et al., 1996).

Using an $f-k$ filter or other filtering procedure does not remove refractions, ground roll, or air waves; it merely suppresses some of their characteristics. When reflection information is not present in the seismic data, the vestiges of refractions, ground roll, and airwaves that invariably remain in the data after filtering, as well as artifacts from wraparound and bandwidth problems, may process smoothly into events that look like reflections. Searching for reflections in some of the raw field files or on frequency-filtered field files is therefore imperative; otherwise, we cannot know with certainty that the data contain legitimate seismic reflections. In every successful shallow reflection survey with which we have been involved, a convincing reflection gleaned from at least one field file or one CMP gather by simple scaling and/or frequency filtering has been identified.

The use of $f$ - $k$ filtering and deconvolution together can generate processing artifacts. Additionally, the disturbance within a data set may be widespread as a result of faulty electronics or other sources of noise, such as lightning. For example, a single electronic noise pulse on one 48-channel record can contaminate 48 bins of a CMP sort. When a data set shows spiky noise on several traces or shots, the deconvolution operator is inverted at the base of its window and scaled proportionally to the amplitude of the spike. The $f-k$ filter process can spread this contamination to other traces via its trace-mixing characteristics. To test whether a data set has this problem, the operator's window length can be varied up and down by a factor of two or three. Theoretically, the deconvolution operation should be most sensitive to reflection wavelets within the autocorrelation window. When the wavelet moves more than a few milliseconds, it is almost certainly a processing artifact rather than a reflector. To continue with our 48-channel, noise-pulse example, a single spike occurring on all traces of a 48-channel record can emerge on a CMP-processed section as a coherent feature 48 traces wide that has the attributes of the filter operators used during processing.

Another type of data contamination resulting from filter operators occurs when clipped data are processed. Clipping can occur inside the geophones, in the seismograph itself, or during processing. In this type of contamination, an artificial, high- frequency content is introduced into the data at the corners of the clipped wavelets. Any subsequent filtering operations will duplicate the filter operator at time samples representing clipped corners. When the resulting filter-operator wavelets in the data happen to stack coherently, they can be (and have been) misinterpreted as reflections.

A processor's choice of automatic gain control (AGC) window length can have an enormous effect on the way a seismic section looks. Displaying the data with an AGC window of $20 \mathrm{~ms}$ as opposed to $200 \mathrm{~ms}$, for example, may alter the look of a seismic section substantially, which can lead to a different set of interpretations. As shown in Figure 9, the AGC window effect produced a high-amplitude zone on the seismic section at times greater than $140 \mathrm{~ms}$ on the section. A longer AGC window (of perhaps $150 \mathrm{~ms}$ ) would have reduced the effect of amplification of the low-signal zone.

Some processing flows have the potential to produce coherent events that may be interpreted erroneously as reflections. In addition to those noted previously, residual statics and dip filters also act as coherency filters. Horizontal stacking, i.e., the mixing or adding of adjacent traces, favors coherency and sets up the other coherency filters to manufacture what appear to be reflectors.

When to stop processing.-Sometimes the brute-stack stage is a good point at which to conclude the data do not contain sufficient reflection energy to merit continuing a project. We assume that we are dealing with genuine reflections only when we see hints of their presence on either the frequency-filtered shot records or on the CMP gathers. In contrast, useful geologic information can be processed into oblivion. For example, Figure 10 indicates the presence of a small bedrock valley by the reversal of apparent dip on the bedrock-reflection hyperbola at times between 55 and $65 \mathrm{~ms}$. Standard CMP processing [without dip moveout (DMO) processing, however] obliterated this bedrock valley from the data. In this instance, the best interpretation would have been achieved by using unprocessed field files.

\section{Data interpretation pitfalls}

When the user is confident that both collection and processing pitfalls have been avoided, the geologic interpretation may

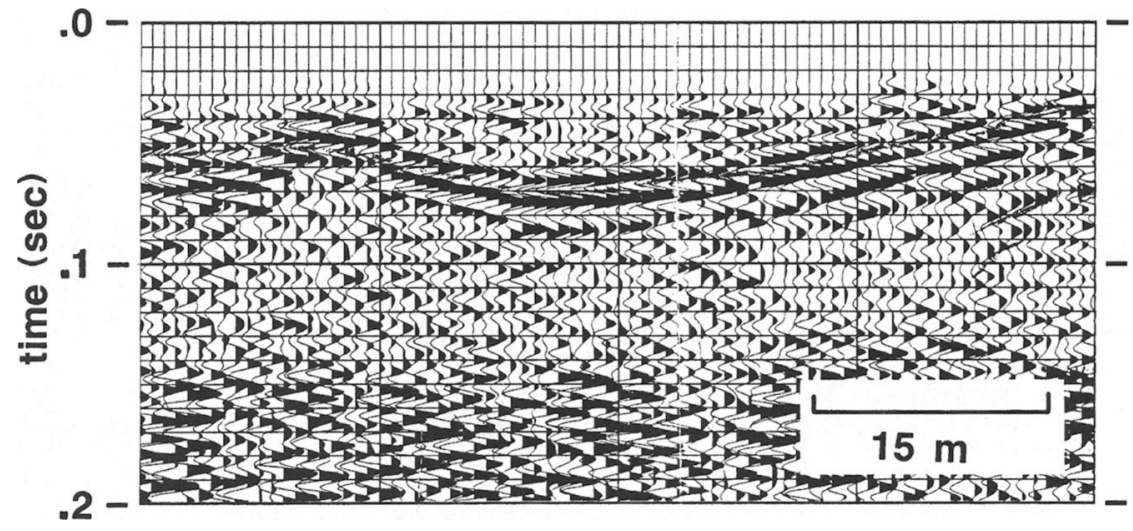

FIG. 9. In this section, choosing an inappropriate automatic gain control window resulted in a relatively quiet zone at times between 90 and $140 \mathrm{~ms}$. The increase in amplitude at $140 \mathrm{~ms}$ is not real; it is a vestige of the AGC window, which causes the ground roll below $140 \mathrm{~ms}$ to be plotted at a larger amplitude. 
begin. Among the main interpretation hazards is the presence of multiple coherent peaks on a seismic section, which may or may not indicate two or more individual reflections. An individual reflector may be represented by one or more peaks, depending upon the shape and bandwidth of the source wavelet and on the acoustic impedance contrast at the individual interfaces.

The wavelets shown in comparisons (Miller et al., 1986,1992) of typical near-surface sources result in two dominant peaks for individual reflections from an interface in which a larger acoustic impedance is encountered, depending somewhat upon display parameters. Conversely, shallow seismic sources typically produce only one dominant peak per reflector when the signal is reflected from an interface characterized by a material having a smaller acoustic impedance.

Deconvolution is not used regularly and is successful only rarely on shallow seismic reflection sections; thus, the effects of source wavelets can remain in the data and pose problems for interpreters. Figure 11 is a schematic of a hypothetical situation (although perhaps oversimplified) in which three reflectors have produced five peaks. Clearly, something must be known about the shape and bandwidth of the source wavelet and about the processing history for the data to be interpreted optimally.
To some extent, interpretation pitfalls can be avoided by judiciously using synthetic seismograms or, at a minimum, by using data from borehole check shots. Synthetic seismograms often can be generated and used to tie reflections to individual geologic units, provided that some well control information and geophysical logs are available. This can be of immense help during a geologic interpretation, which in most cases must

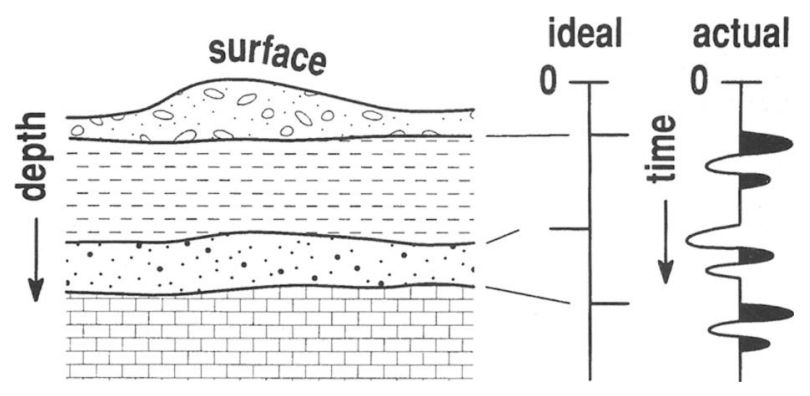

FIG. 11. A hypothetical geologic, acoustic-impedance, and synthetic-seismogram model showing reversed polarity at the second reflective interface. In such a case, shallow reflection data commonly produce five peaks for only three interfaces.

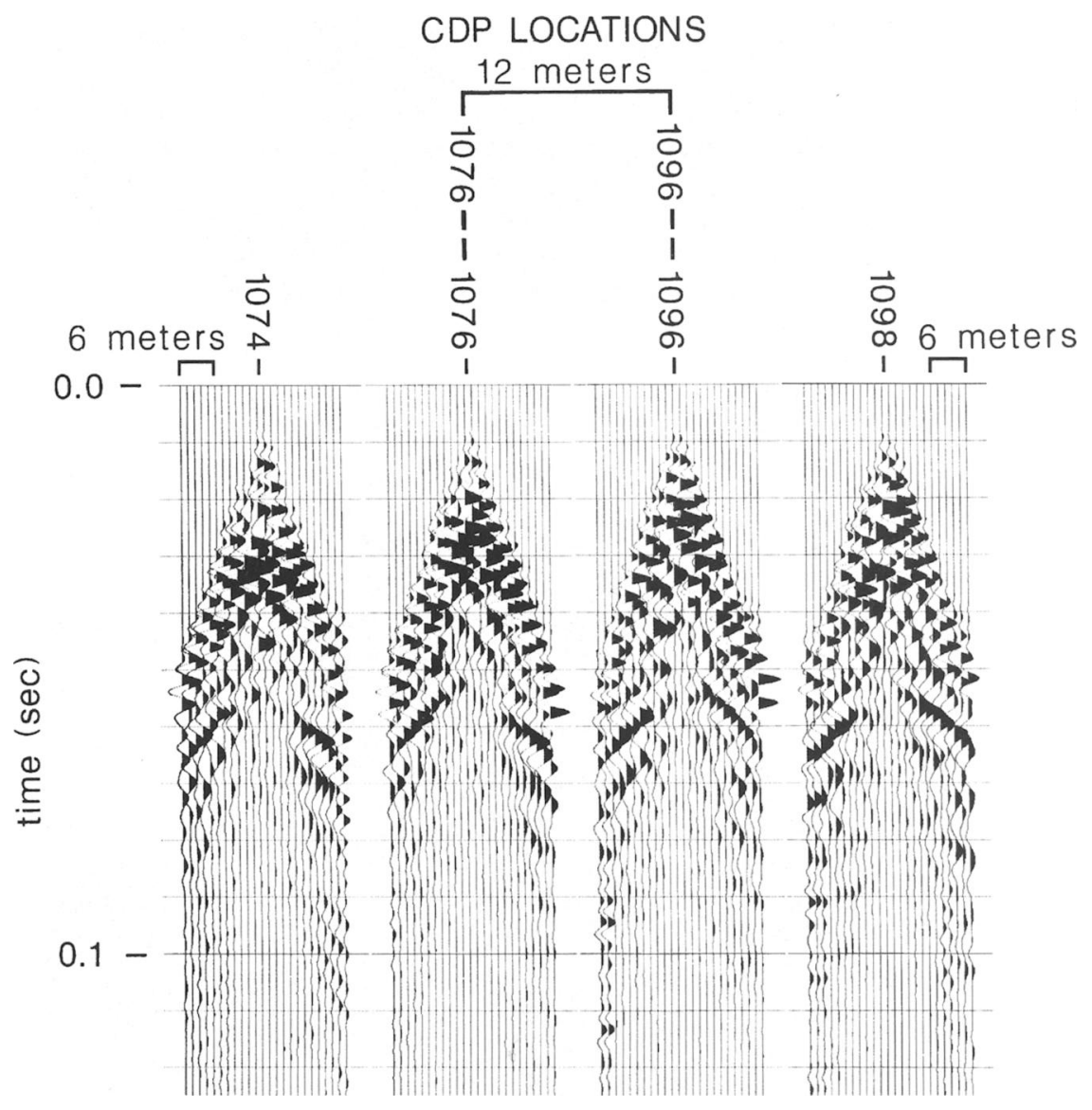

FIG. 10. Bedrock valley interpreted from field files. Note the asymmetry of the reflector at times between 55 and 65 ms. At locations 1074 and 1076, the apex of the reflection hyperbola is to the left of the shotpoint, which indicates the updip direction is to the left. At locations 1096 and 1098, the apex of the reflection hyperbola is to the right of the shotpoint, indicating the updip direction is to the right. Consequently, we know that a bedrock valley is present within the $12-\mathrm{m}$ horizontal distance between locations 1076 and 1096. This bedrock valley disappeared during processing. 
be tested by other methods, including corroborative drilling at critical spots.

Data processing pitfalls and interpretation problems are not unrelated. For example, an interpretation can depend heavily on the velocity function used. Figures 12 and 13 (Steeples and Miller, 1990) show how a refined velocity function can affect interpretation when a small horst has been superimposed on a gently sloping interface. Figure 14 (Miller, 1992) illustrates the sensitivity of data quality to small changes in NMO stretch mute. Note that $\mathrm{S} / \mathrm{N}$ ratio can be an important variable in the interpretation of a seismic section.

Incorrect or uncompensated static corrections.-Early or delayed arrivals of seismic energy, called statics, result from a laterally nonuniform, near-surface velocity or elevation. They are a problem for both conventional and shallow reflection surveys. The severity of the problem, however, is much more significant during shallow surveys when the total static correction is a significant fraction of the two-way reflection time. Because the data contain high frequencies, the static corrections may be more than the dominant periods of the reflection wavelets.

Managing the statics problem with processing routines can be attempted with some degree of confidence when a large number of reflections are present. Unfortunately, the number of total reflection events on shallow data sets tends to be low, which sets the stage for the production of artifacts during efforts to compensate for these time irregularities. In fact, correlation statics routines have been quite successful in removing real structures, generating bogus structures, and producing coherent events from noise.

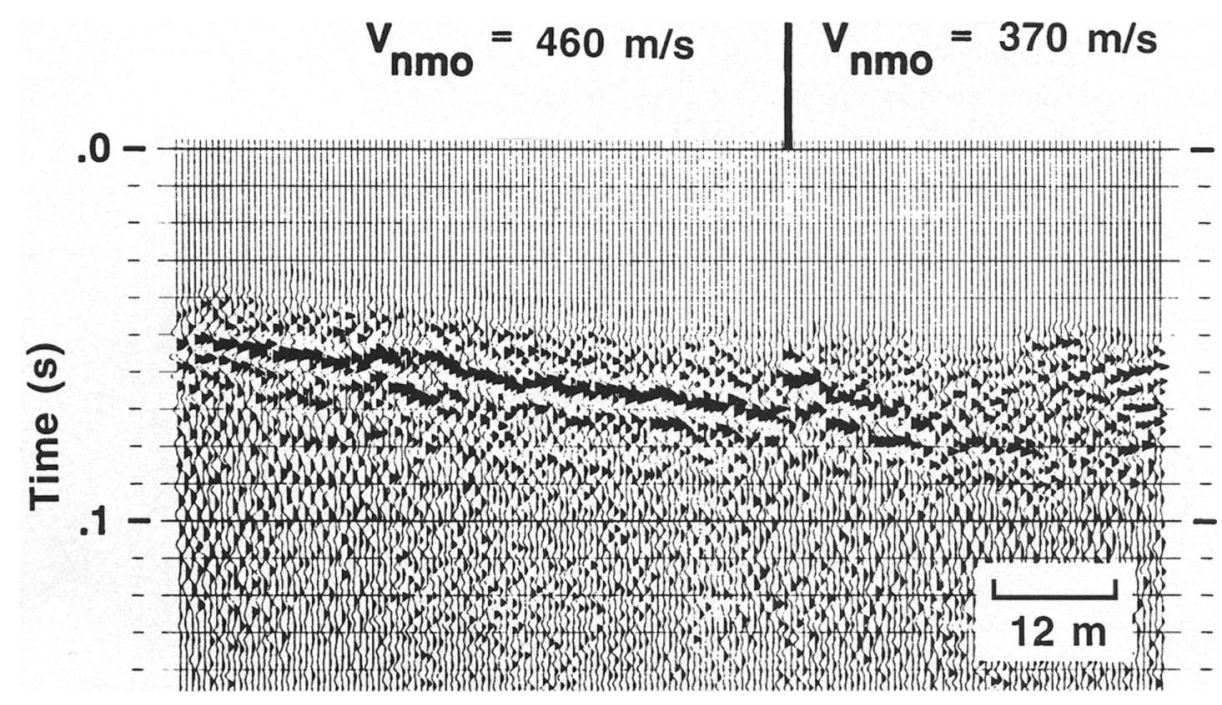

FIG. 12. The effect of incorrect stacking velocity on a shallow reflector. The apparent fault to the right of the center of the seismic section is not a fault but is the effect of incorrect stacking velocity. Compare with Figure 13, in which a more appropriate velocity function was used (from Steeples and Miller, 1990).

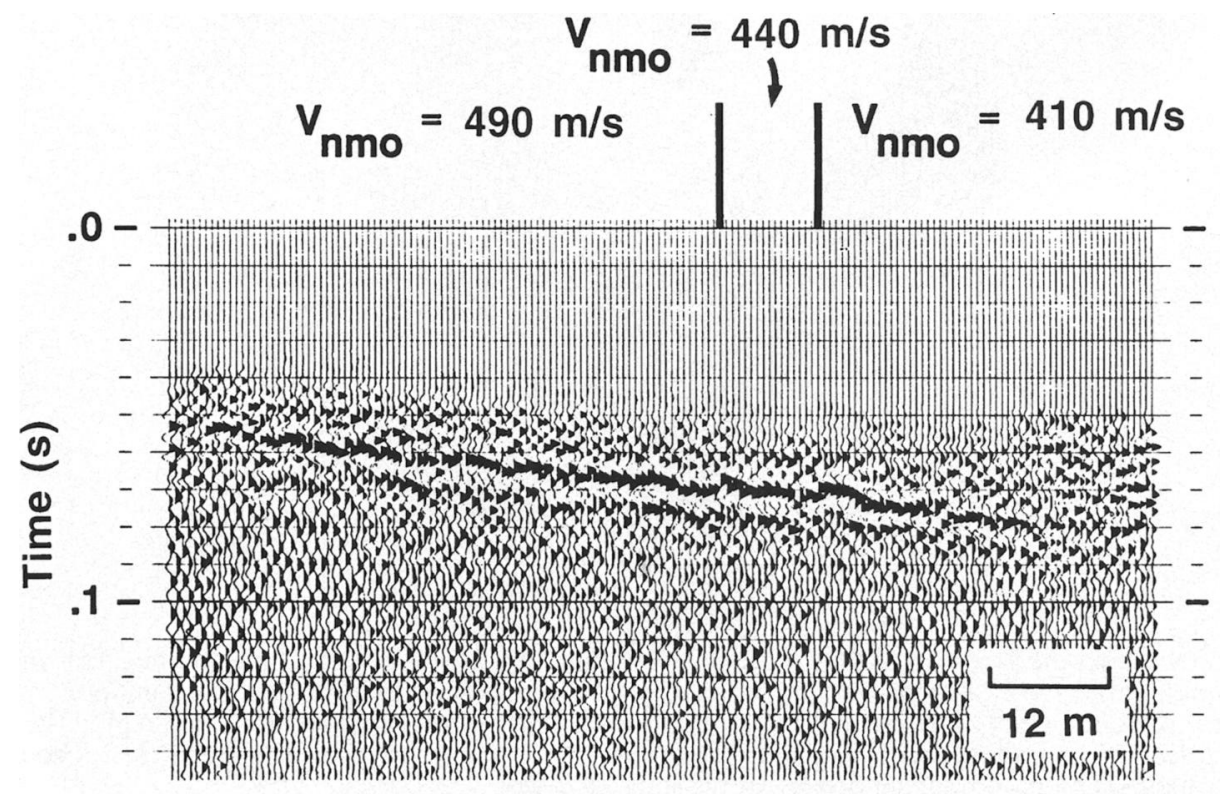

FIG. 13. The same data as those in Figure 12 but with a better stacking velocity function. No faulting is evident (from Steeples and Miller, 1990). 
Probably the most effective method of correcting shallow reflection data for near-surface static irregularities was developed in association with the optimum-window method of Hunter et al. (1984). Most static variations originate above the water table; thus, when a water table reflection can be identified, most statics can be compensated by flattening the reflection. Unfortunately, in shallow reflection data, we have seen that strong

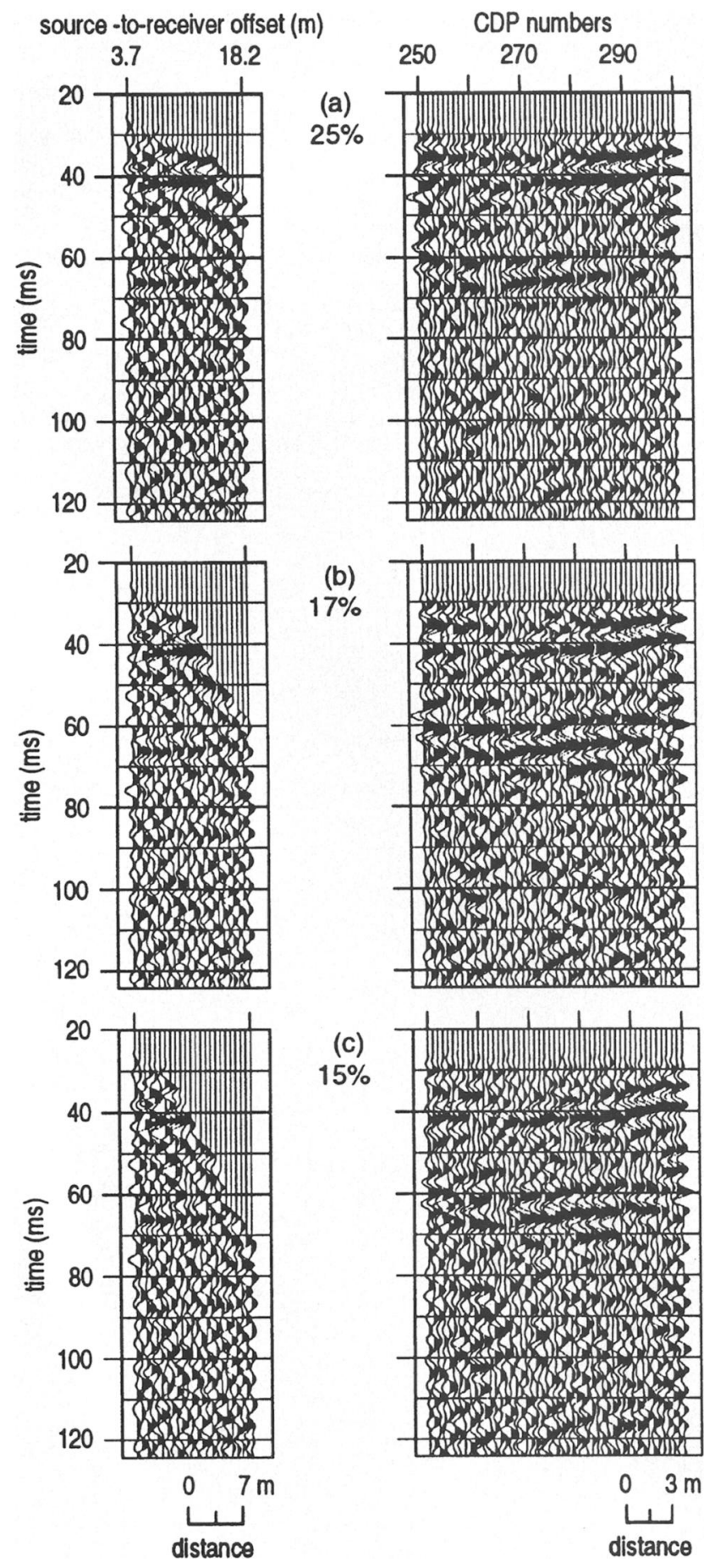

FIG. 14. The first-arrival stretch mute is not a particularly important parameter in deep-reflection surveying. In near-surface surveys, however, it can be significant (data from Miller, 1992). water table reflections are not common. This leaves refraction statics, common-offset statics, or correlation statics routines as options.

Correlation statics routines are widely used, but they are also very susceptible to the generation of artifacts. The main source of error comes from excessive allowable shifts. As the dominant frequency of the reflection wavelet increases, the amount of shift that can be applied without skipping a cycle decreases. For example, consider a reflection-time event at $40 \mathrm{~ms}$ having $2 \mathrm{~ms}$ of static relative to adjacent traces. This static represents $1 / 5 \lambda$ of a $100-\mathrm{Hz}$ reflection; but if the dominant frequency is $500 \mathrm{~Hz}$, it will be a full-wavelength static. Depending on the bandwidth of the reflection wavelet, it is very unlikely that this static can be managed with correlation statics routines alone. Limiting the maximum allowable static shift in correlation statics routines to one-quarter of the dominant wavelet period and ensuring that at least two (and preferably more) reflection events are within the correlation window minimizes the chance of generating static artifacts. Clearly, maximizing frequency is our ultimate goal; but regardless of how static correction is accomplished, compensating for actual static anomalies becomes more difficult as frequency increases.

Low fold on some geophones.-Sometimes recording geometry, optimum recording window, and depth to reflector can combine in such a way that the multiplicity or CMP fold on the processed data can vary greatly with time within a particular seismic section. When the midpoint is the same for 12 offset distances, for example, a stack is referred to as 12-fold. But when a shallow reflector exists under low-fold circumstances and full fold is 12 , calling the data for the whole section " 12 -fold" could be misleading.

In some cases, the fold level may drop so much that the data we see on the shallowest parts of a section may be equivalent to those we see on a common-offset section. When the $\mathrm{S} / \mathrm{N}$ ratio is sufficiently high, plotting common-offset sections with and without NMO corrections for one or more of the near-trace offsets can be advantageous following the application of statics and filtering. Determining whether any of the common-offset sections resembles the final stacked section can be helpful when interpreting CMP sections. Pullan and Hunter (1985) show that substantial distortion of the reflected wavelet can occur at wide incidence angles with shallow reflections. The common-offset sections from one or more of the closer offsets may provide important information about reflected wavelets that have not been distorted by NMO corrections or by wide angles of incidence. Care should be taken that close-offset, single traces are not corrupted by overfiltering, which can produce what appear to be high-frequency reflection wavelets.

\section{CONCLUSIONS}

Shallow seismic reflection has reached an important juncture as a potentially practicable and reliable method of profiling the near surface of the earth. The real cost per channel of the modern seismograph has decreased strikingly in recent years, as has the cost of related data processing hardware and software. An increase in the accessibility of these items has produced a concomitant increase in interest in the shallow seismic reflection profiling method, with many new environmental and geophysical applications being developed as a result. However, all too 
often projects succumb to one or more of the pitfalls discussed here, thus damaging the overall reputation of the technique.

As a result, practitioners of the method must be knowledgeable in both theory and practice when applying shallow seismic techniques and must be exceptionally careful not to misinterpret as reflections the many coherent waves that are not reflections. This is particularly true of the types of wave coherencies that develop during or that can be enhanced by digital processing. Some of the pitfalls an interpreter may face when dealing with near-surface data include the spatial aliasing of ground roll, interpreting processed airwaves as reflected seismic waves, misinterpreting refractions as reflections on stacked CMP sections, and failing to recognize processing artifacts. Therefore, particular care must be taken during all phases of a project, which includes planning and modeling as well as data collection, processing, and interpretation. Finally, to validate their conclusions, interpreters must have access to at least one field file, along with display copies of one or more of the intermediate processing steps whenever possible.

\section{ACKNOWLEDGMENTS}

Reviews by Susan Pullan, Brad Carr, and Joel Watkins greatly helped the focus and readability of this paper. Editorial assistance by Lee Blackledge during several drafts and preparation of figures by Pat Acker are greatly appreciated.

\section{REFERENCES}

Evison, F. F., 1952, The inadequacy of the standard seismic techniques for shallow surveying: Geophysics, 17, 867-875.

Hoover, G. M., and O'Brien, J. T., 1980, The influence of the planted geophone on seismic land data: Geophysics, 45, 1239-1253.

House, J. R., Boyd, T. M., and Haeni, F. P., 1996, Haddam Meadows, CT: A case study for the acquisition, processing, and relevance of 3-D seismic data as applied to the remediation of DNAPL contamination, in Weimer, P., and Davis, T. L., Eds., Applications of 3-D seismic data to exploration and production: AAPG Studies in Geology 42, Am. Assn. Petr. Geol., 257-265.

Hunter, J. A., Pullan, S. E., Burns, R. A., Gagne, R. M., and Good, R. S., 1984, Shallow seismic reflection mapping of the overburdenbedrock interface with the engineering seismograph-some simple techniques: Geophysics, 49, 1381-1385.

Keiswetter, D. A., 1995, Seismic reflection analysis of the Manson impact structure, Iowa; Ph.D. dissertation, Univ. of Kansas.

Krohn, C. E., 1984, Geophone ground coupling: Geophysics, 49, 722 731.

Miller, R. D., 1992, Normal moveout stretch mute on shallow-reflection data: Geophysics, 57, 1502-1507.

Miller, R. D., Pullan, S. E., Steeples, D. W., and Hunter, J. A., 1992, Field comparison of shallow seismic sources near Chino, California: Geophysics, 57, 693-709.

Miller, R. D., Pullan, S. E., Waldner, J. S., and Haeni, F. P., 1986, Field comparison of shallow seismic sources: Geophysics, 51, 2067-2092.

Pullan, S. E., and Hunter, J. A, 1985, Seismic model studies of the overburden-bedrock problem: Geophysics, 50, 1684-1688.

Steeples, D. W., and Miller, R. D., 1990, Seismic-reflection methods applied to engineering, environmental, and ground-water problems, in Ward, S., Ed., Review and tutorial: Investigations in geophysics 5 , Soc. Expl, Geophys., 1-30.

Yilmaz, O., 1987, Seismic data processing: Investigations in geophysics 2, Soc. Expl. Geophys. 